\title{
LA MOSKITIA HONDUREÑA, EL LÍMITE MÁS AL NORTE DE LA DISTRIBUCIÓN ACTUAL DEL OSO HORMIGUERO GIGANTE (Myrmecophaga tridactyla)
}

\author{
Héctor Orlando Portillo Reyes* \\ 1 *Fundación de Ciencias para el Estudio y la Con- \\ servación de la Biodiversidad (INCEBIO) Tegucigalpa, \\ Honduras
}

Autor de correspondencia: Héctor Orlando Portillo:

hectorportilloreyes@gmail.com

\section{RESUMEN}

El oso hormiguero gigante también conocido en Centro América como oso caballo (Myrmecophaga tridactyla), es una de las especies carismáticas del mundo. Se ha documentado en la literatura que los límites de la distribución más septentrional para esta especie son Belice y Guatemala. El mapa de su distribución para Centro América da inicio en toda la franja Caribe de Panamá e incluye todo el territorio de Costa Rica, Nicaragua, Honduras, El Salvador y una mínima porción de la costa del Caribe de Guatemala y Belice. Sin embargo, M. tridactyla no se registra en los últimos 100 años para Guatemala, Belice y El Salvador. En Honduras se realizaron diferentes monitoreos en la región Caribe y Moskitia registrándose fotocapturas del oso caballo únicamente en la Reserva de la Biosfera del Río Plátano y la Reserva propuesta de Rus Rus. Este análisis propone como límite septentrional de la distribución del M. tridactyla la región de la Moskitia hondureña, basados en la ausencia de esta especie en los listados actuales oficiales de los países de Guatemala, El Salvador y Belice, en la revisión bibliográfica para Centro América y en los monitoreos que se realizaron en la región Caribe y Moskitia hondureña.

Palabras clave: Caribe, Centro América, Moskitia, oso caballo, septentrional.

\section{ABSTRACT}

The giant anteater also known in Central America as oso caballo (Myrmecophaga. tridactyla), is one of the world's charismatic species. It has been mentioned for different sources that giant anteater most northern limit distributions are Belize and Guatemala. The distribution map for Central America begins and extends throughout the Caribbean of Panama and includes the entire territory of Costa Rica, Nicaragua, Honduras, El Salvador and a minimal portion of the Caribbean coast of Guatemala and Belize. However M. tridactyla is not recorded in the last 100 years in Guatemala, Belize and El Salvador. In Honduras several biological monitoring were performed in 
the Caribbean and the Moskitia region recorded evidence for the specie only for the Biosphere Reserve of Río Plátano and the biological Reserve of Rus Rus in the Moskitia region. This analysis propose as most northerly for $M$. tridactyla the Honduran Moskitia region, based in the absence on the most recent check list for Guatemala, El Salvador, and Belize, also literature review for Central America and the biological monitoring performed in the Caribbean and Moskitia region.

Keywords: Caribbean, Central America, giant anteater, Moskitia.

\section{INTRODUCCIÓN}

Se menciona que los límites de la distribución más al norte para el oso caballo (Myrmecophaga tridactyla) ha sido Belice y Guatemala. Se extiende hacia el resto de Centro América, con límites de distribución en Sur América, específicamente en Bolivia y el norte de Argentina. (Hall, 1981; Wetzel, 1982, 1985; Gardner, 1993; Emmons, 1997; Reid, 1997; Nowak, 1999; McCain, 2001). Mientras que en Uruguay se considera extinto (Porini et al., 2004). Los registros para Belice fueron hechos por Alston (1879-1882) en Punta Gorda, y en el sureste de Guatemala por Handley (1950). Según McCain (2001) el registro de Punta Gorda fue basado en un reporte sin documentar de Alston. Algunos autores mencionan que el oso caballo estaba extirpado de la región mesoamericana (Timm and Laval, 2002); sin embargo, existen registros basados en huellas, huesos, cráneos, avistamientos, videos y fotografías que evidencian su presencia en la región de la Moskitia de Nicaragua y Honduras (Koster, 2008; Castañeda, 2008b; Portillo et al., 2010; Gonthier y Castañeda, 2013). Para la Unión Internacional para la Conservación de la Naturaleza (UICN, 2004) el oso caballo (M. tridactyla) se encuentra en la categoría de Vulnerable, para Honduras se encuentra en lista de preocupación según resolución GG-DAPVS-003-98 AFE/COHDEFOR (en literatura).
El oso hormiguero gigante, $(M$. tridactyla) es una de las especies carismáticas del mundo (Diniz y Brito, 2012). Esta especie es inconfundible, posee una trompa tubular larga. De piel gruesa y áspera y pelo largo. Su cola posee un pelaje largo, en algunos individuos sobrepasa la longitud del cuerpo. En ambos lados del cuerpo se marca una franja oscura bordada que se extiende desde la garganta, pecho y oblicuamente abarca los flancos del cuerpo hasta el dorso (Marineros y Martínez, 1998; Figura 2 y 3).

Según Porini et al. (2004) el mapa de distribución del M. tridactyla para Centro América se extiende geográficamente de manera homogénea en la franja Caribe de Panamá, todo el territorio de Costa Rica, Nicaragua, Honduras y El Salvador (Figura 1). Sin embargo, el oso caballo no es incluido en los listados actualizados de los mamíferos de Belice, Guatemala y El Salvador (McCarthy, 1998; McCarthy et al., 2006; Owens y Girón, 2012).

En Honduras los registros históricos de Goodwin (1942) no lo mencionan, no obstante comenta su posible presencia en la región de las Segovia, frontera entre Honduras y Nicaragua. Marineros y Martínez (1998), recopilan avistamientos de oso caballo hecho por personas locales, estudiantes y biólogos en campo para la región Caribe y Moskitia. Durante el periodo de 20002005 y bajo el programa de Monitoreo Biológico realizado en 24 áreas prote- 


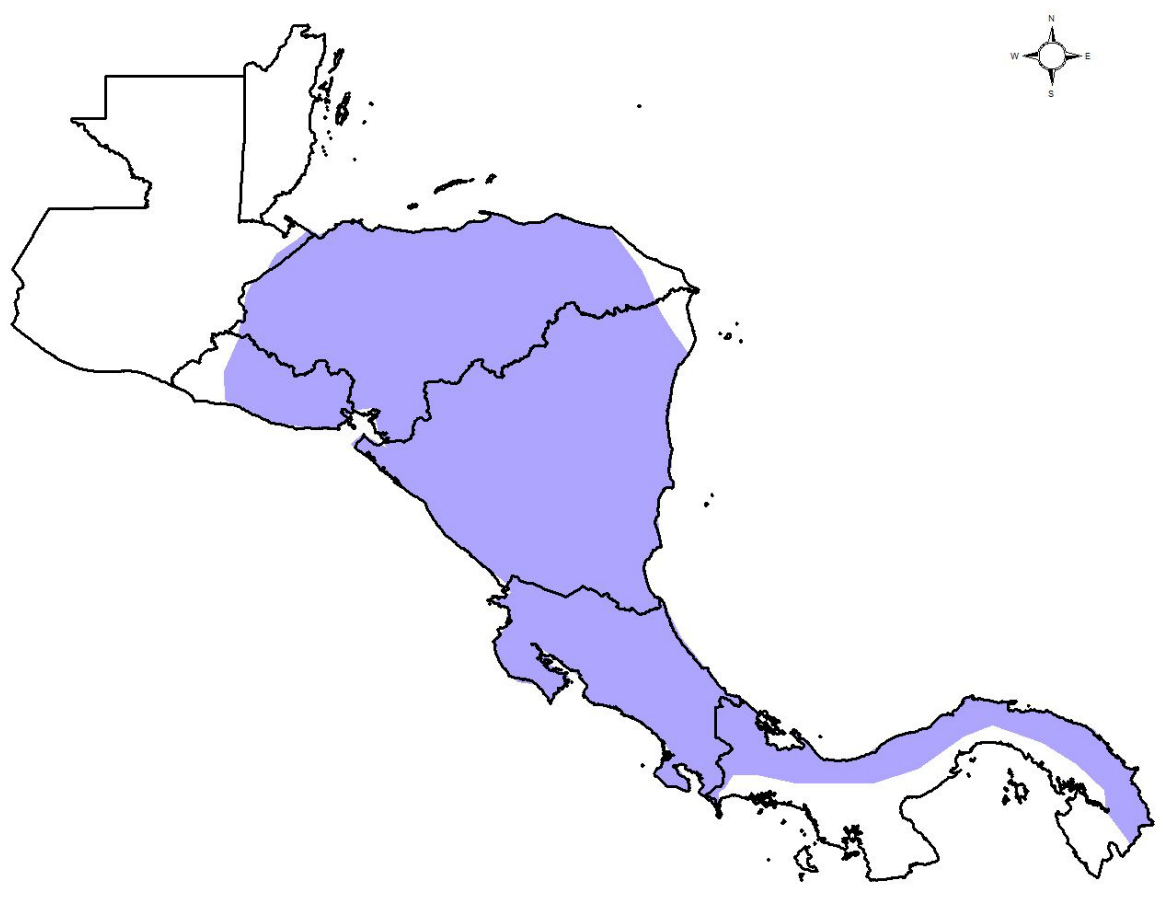

Figura 1. Mapa propuesto por Porini et. al., 2004, en donde menciona que la distribución septentrional del Myrmecophaga tridactyla incluye una pequeña franja en la parte fronteriza del Caribe de Guatemala y Honduras. El mapa asume en Honduras, El Salvador, Nicaragua, Costa Rica distribución en casi homogénea en cada uno de estos países y en Panamá una franja que cubre la parte media hacia el Caribe de Panamá (Mapa elaborado por el autor).

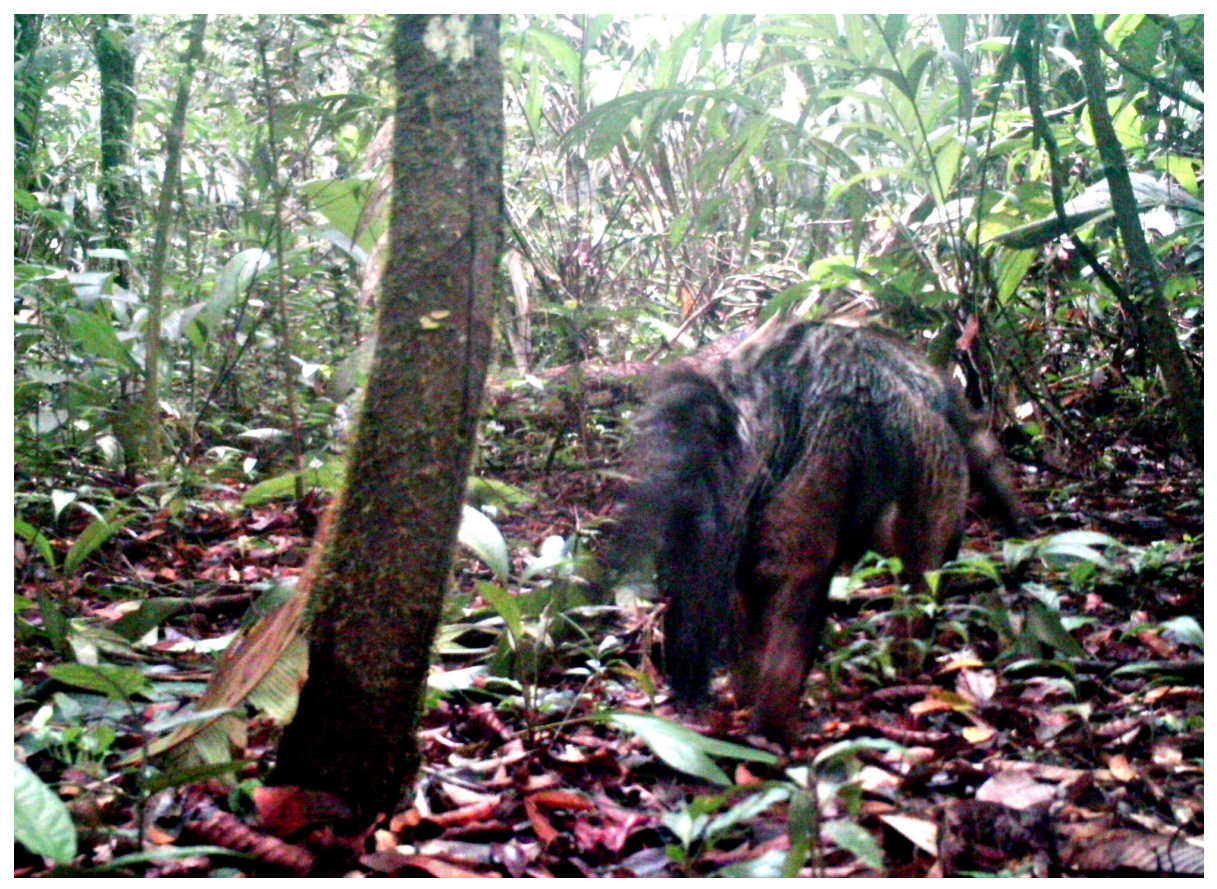

Figura 2. Fotocaptura de oso caballo (M.tridactyla) en los bosques riparios en transición a las sabanas de pino en Mabita, Rus Rus para el año 2012 (cortesía del proyecto Moskitia/PNUD). 


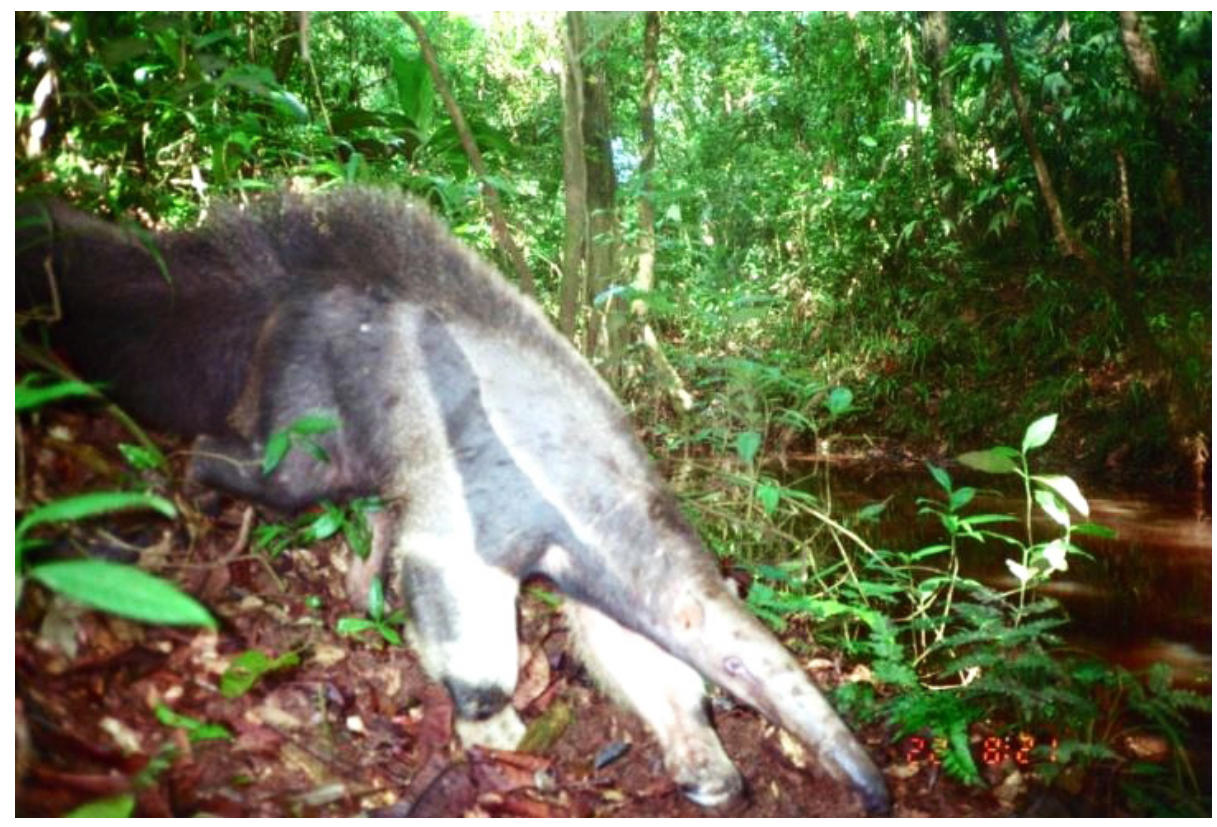

Figura 3. Fotocaptura de oso caballo ( $M$. tridactyla) en los bosques riparios del Río Sigre, Biosfera del Río Plátano. (Fotografía de trampa cámara, Franklin Castañeda, 2009).

gidas del Caribe, Centro y Moskitia de Honduras donde se asume que el oso caballo puede estar extirpado de la región Caribe tal como lo menciona Portillo et al., 2010, siendo únicamente la región de al Moskitia hondureña el hábitat potencial de su distribución (Portillo et al., 2010; Gonthier y Castañeda, 2013).

El objetivo de este análisis es proponer como límite más septentrional del M. tridactyla la región de la Moskitia hondureña y su distribución potencial, mostrando que no hay para Honduras una distribución homogénea tal y como lo menciona Porini et al. (2004), así como la no detección de la especie durante el monitoreo de trampas cámara en el Caribe hondureño.

\section{METODOLOGÍA}

\section{Sitio del Estudio}

La región de la Moskitia se localiza a $15^{\circ} 20^{\prime} 00^{\prime \prime}$ latitud Norte y $84^{\circ} 83^{\prime}$ $00^{\prime \prime}$ longitud Oeste. El tipo de vegetación se compone de bosque húmedo tropical (bh-T) y el bosque muy húmedo subtropical (bmh-ST) con rangos altitudinales de 10-800 msnm, con predominancia de los ecosistemas del bosque latifoliado de tierras bajas y las sabanas de Pinus caribaea (Mejía y House, 2002). La temperatura máximas promedio es de $30.2^{\circ} \mathrm{C}$, y rangos de precipitación anual entre 1,500 y 3,100 mm, con una humedad relativa que oscila entre el 74 y $82 \%$.

Esta región abarca una extensión territorial de aproximadamente $1,578,470$ (ha) (DAPVs, 2005). Para este análisis la región actual de la Moskitia es la masa continua de bosque que se extiende desde el área protegida de La Reserva de la Biosfera (RBio) del Río Plátano, La RBio Tawahka Asagni, El Parque Nacional (PN) Patuca, Las áreas del territorio Indígena de Rus Rus, Warunta y Mocorón. Estos se encuentran en los departamentos de Colón, Gracias a Dios, Olancho y El Paraíso.

La región del Caribe hondureño se localiza entre $15^{\circ} 20^{\prime} 00^{\prime \prime}$ latitud Norte y $85^{\circ} 80^{\prime} 00^{\prime \prime}$ longitud Oeste. El 
Tabla 1. Áreas protegidas de la región del Caribe y Moskitia donde se realizó monitoreo biológico usando trampas cámara.

\begin{tabular}{|c|c|c|}
\hline ÁREA DE MONITOREO & PROYECTO & REFERENCIA \\
\hline $\begin{array}{l}\text { Parque Nacional Pico Bonito } \\
\text { (Caribe) }\end{array}$ & $\begin{array}{l}\text { MIRA/USAID } \\
\text { Proyecto Panthera }\end{array}$ & $\begin{array}{l}\text { Portillo et al., } 2006 \\
\text { Castañeda, F. } 2010\end{array}$ \\
\hline $\begin{array}{l}\text { Parque Nacional Sierra de Agalta } \\
\text { (Moskitia) }\end{array}$ & MIRA/USAID & Estrada N. 2006 \\
\hline Biosfera del Río Plátano (Moskitia) & $\begin{array}{l}\text { Mejorando Nuestra Herencia/ } \\
\text { UNESCO } \\
\text { Wildlife Conservation Society } \\
\text { (wCS) } \\
\text { Proyecto Panthera }\end{array}$ & $\begin{array}{l}\text { Castañeda, F. } 2007 \\
\text { Castañeda, F. } 2008 \text { b } \\
\text { Castañeda, F. 2008a } \\
\text { Castañeda } 2010\end{array}$ \\
\hline $\begin{array}{l}\text { Área propuesta de Rus Rus } \\
\text { (Moskitia) }\end{array}$ & $\begin{array}{l}\text { WCS } \\
\text { Proyecto Moskitia/PNUD }\end{array}$ & $\begin{array}{l}\text { Portillo et al., } 2008 \\
\text { Portillo, H. } 2013\end{array}$ \\
\hline $\begin{array}{l}\text { Reserva de la Biosfera Tawahaka } \\
\text { (Moskitia) }\end{array}$ & WCS & Portillo y Vásquez 2009 \\
\hline $\begin{array}{l}\text { Parque Nacional Jeannette Kawas } \\
\text { (Caribe) }\end{array}$ & Proyecto Panthera & $\begin{array}{l}\text { Castañeda et al., 2013a } \\
\text { Castañeda et al., 2013b }\end{array}$ \\
\hline $\begin{array}{l}\text { Refugio de Vida Silvestre de } \\
\text { Texiguat (Caribe) }\end{array}$ & Proyecto Procorredor & ESNACIFOR, 2012 \\
\hline $\begin{array}{l}\text { Jardín Botánico de Lancetilla } \\
\text { (Caribe) }\end{array}$ & Proyecto Procorredor & ESNACIFOR, 2012 \\
\hline $\begin{array}{l}\text { Refugio de Vida Silvestre de Cuero } \\
\text { y Salado (Caribe) }\end{array}$ & $\begin{array}{l}\text { Proyecto RECOTURH } \\
\text { Proyecto CANATURH }\end{array}$ & Portillo, H. 2013 \\
\hline Parque Nacional Patuca (Moskitia) & $\begin{array}{l}\text { Proyecto Panthera } \\
\text { Helvetas-Honduras } \\
\text { Asociación Patuca }\end{array}$ & Castañeda et al., 2013 \\
\hline $\begin{array}{l}\text { Parque Nacional Nombre de Dios } \\
\text { (Caribe) }\end{array}$ & Proyecto Panthera & Castañeda, F. 2012 \\
\hline $\begin{array}{l}\text { Parque Nacional Capiro y } \\
\text { Calentura (Caribe) }\end{array}$ & $\begin{array}{l}\text { Centro Universitario del } \\
\text { Litoral Atlántico }\end{array}$ & $\begin{array}{l}\text { Flores, A } 2012 \text { (doc no } \\
\text { publicado) }\end{array}$ \\
\hline $\begin{array}{l}\text { Parque Nacional Sierra de Río } \\
\text { Tinto (Moskitia) }\end{array}$ & Proyecto Ecosistemas/PNUD & $\begin{array}{l}\text { Responsable ICF (doc no } \\
\text { publicado) }\end{array}$ \\
\hline
\end{tabular}

tipo de vegetación se compone de bosque húmedo tropical (bh-T) y el bosque muy húmedo subtropical (bmh-ST) con rangos altitudinales de 10-2000 msnm, con predominancia de los ecosistemas del bosque latifoliado de tierras bajas, bosques de transición a pino $(P$. caribaea) y de bosques de pino a bosques nublados (Mejía y House 2002). La temperatura máximas promedio es de $27^{\circ} \mathrm{C}$, y rangos de precipitación promedio anual es de 2,885 $\mathrm{mm}$ con una humedad relativa que oscila entre el 83 y $86 \%$. Para este análisis la región del Caribe lo comprenden las áreas protegidas de: PN Jeannette Kawas, Refugio de Vida Silvestre (RVS) de Texiguat, PN Pico Bonito, Jardín Botánico de Lancetilla, RVS de Cuero y Salado, PN Nombre de Dios y el PN Capiro y Ca- 
Tabla 2. Localidad y año del registro de oso caballo (M. tridactyla) en la región Caribe y Moskitia hondureña.

\begin{tabular}{|c|c|c|c|c|c|}
\hline No & Localidad & Latitud & Longitud & Evidencia de registro & Año de registro \\
\hline 1 & *Las Marías & 15.78 & -84.78 & Fotografía & 1996 \\
\hline 2 & + San Alejo & 15.43 & -87.34 & Avistamiento & 1978 \\
\hline 3 & +Santa Rosa de Aguan & 15.56 & -85.40 & Avistamiento & 1998 \\
\hline 4 & +Isletas & 15.36 & -86.12 & Avistamiento & 1998 \\
\hline 5 & +Susmay & 14.56 & -86.05 & Avistamiento & 1998 \\
\hline 6 & +Tusi-cocal & 15.83 & -84.52 & Huellas & 1998 \\
\hline 7 & +Krautara & 15.03 & -84.93 & Huellas & 1998 \\
\hline 8 & +Krausirpe & 15.06 & -84.92 & Huellas & 1998 \\
\hline 9 & + Picholón & 14.54 & -85.2 & Huellas & 2005 \\
\hline 10 & $\varnothing$ Rus Rus & 14.72 & -84.46 & Avistamiento & 2007 \\
\hline 11 & $\varnothing$ Rus Rus & 14.74 & -84.47 & Avistamiento & 2007 \\
\hline 12 & Ø Tapalwas & 14.98 & -84.58 & Video & 2008 \\
\hline 13 & $\varnothing$ Tapalwas & 14.05 & -84.53 & Huellas & 2008 \\
\hline 14 & $\varnothing$ Tapalwas & 15.05 & -84.46 & Huellas & 2008 \\
\hline 15 & $\varnothing$ Auka & 14.93 & -83.83 & Avistamiento & 2008 \\
\hline 16 & ®Río Sigre & 15.52 & -84.66 & Fotocaptura & 2008 \\
\hline 17 & (RRío Sigre & 15.48 & -84.7 & Fotocaptura & 2008 \\
\hline 18 & ®Río Sigre & 15.56 & -84.63 & Fotocaptura & 2008 \\
\hline 19 & ®Río Sigre & 15.49 & -84.71 & Fotocaptura & 2008 \\
\hline 20 & \Mabita & 14.84 & -84.49 & Fotocaptura & 2012 \\
\hline 21 & \Mabita & 14.85 & -84.51 & Fotocaptura & 2012 \\
\hline
\end{tabular}

\section{Fuente:}

* McCain, K .1996.

+ Marineros y Martínez 1998.

† Comunicación personal (Mario Guifarro)

® Castañeda, F. 2008b (puntos al azar dentro del rango del polígono de $113 \mathrm{~km}^{2}$ del área total del estudio)

$\varnothing$ Portillo et al., 2010

\ Portillo, H. 2012 


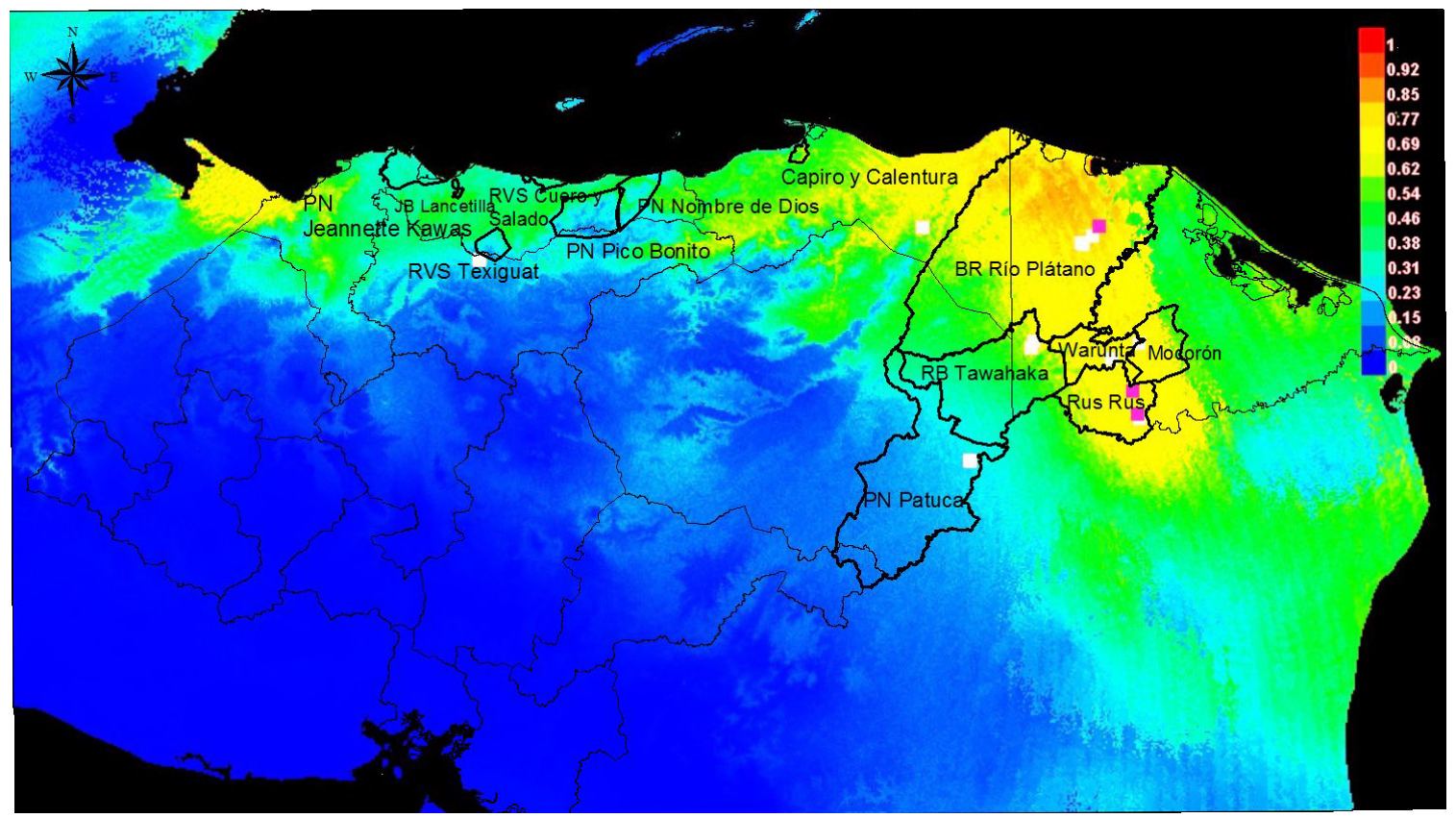

Figura 4. Mapa de modelación de los registros de la presencia de oso caballo $(M$. tridactyla) en la región de la Moskitia hondureña, como puede observarse los sitios de color amarillo modelan condiciones adecuadas para el hábitat del oso caballo, tal como lo evidencia su registro en los sitios, en la Reserva de la Biosfera (RBIO) Río Plátano, Parque Nacional Warunta, Area de Territorio Indígena Mocorón, y una pequeña porción de Reserva de la Biosfera Tawahka Asagni. El color verde representan condiciones aceptables y típicas para $M$. tridactyla, las sombras tenues de azul claro al azul oscuro indican una baja probabilidad de tener las condiciones de hábitat adecuadas para la especie.

lentura, en los departamentos de Cortes, Atlántida, Yoro y Colón.

\section{Muestreo}

Para los años 2006-2014, se instalaron trampas cámara en áreas protegidas de la región Caribe y Moskitia como parte del monitoreo de jaguares y otras especies de mamíferos terrestres utilizando el protocolo propuesto por Silver (2003), realizado por las siguientes instituciones y proyectos (Tabla 1).

El monitoreo en la región Caribe y Moskitia tomo un esfuerzo de muestreo aproximado de 18,721 noches cámara de los cuales 8,757 noches cámara se registraron para las áreas protegidas del Caribe hondureño y 9,964 noches cámara para la región de la Moskitia, con un número aproximado de 1,000 cámaras distribuidas en la región del Caribe y la Moskitia hondureña. Estas cámaras fueron colocadas en la zona núcleo y zona de amortiguamiento de cada una de las áreas protegidas muestreadas.

\section{Análisis de los registros}

Para la elaboración de la base de datos referente a los registros de oso caballo en Honduras, se revisaron diversas fuentes. Para los datos históricos se consultó a Marineros y Martínez (1998); para los datos más recientes se consultó los datos del monitoreo biológico del proyecto de Biodiversidad en Áreas Protegidas (PROBAP/AFE-COHDEFOR, 2005), los informes técnicos de Castañeda 2008a; Castañeda 2008b, Portillo 2013, Portillo et al., 2010 y Gonthier y Castañeda 2013 (Tabla 2). 
Sensibilidad vs 1- Especificidad para Myrmecophaga tridactyla 0

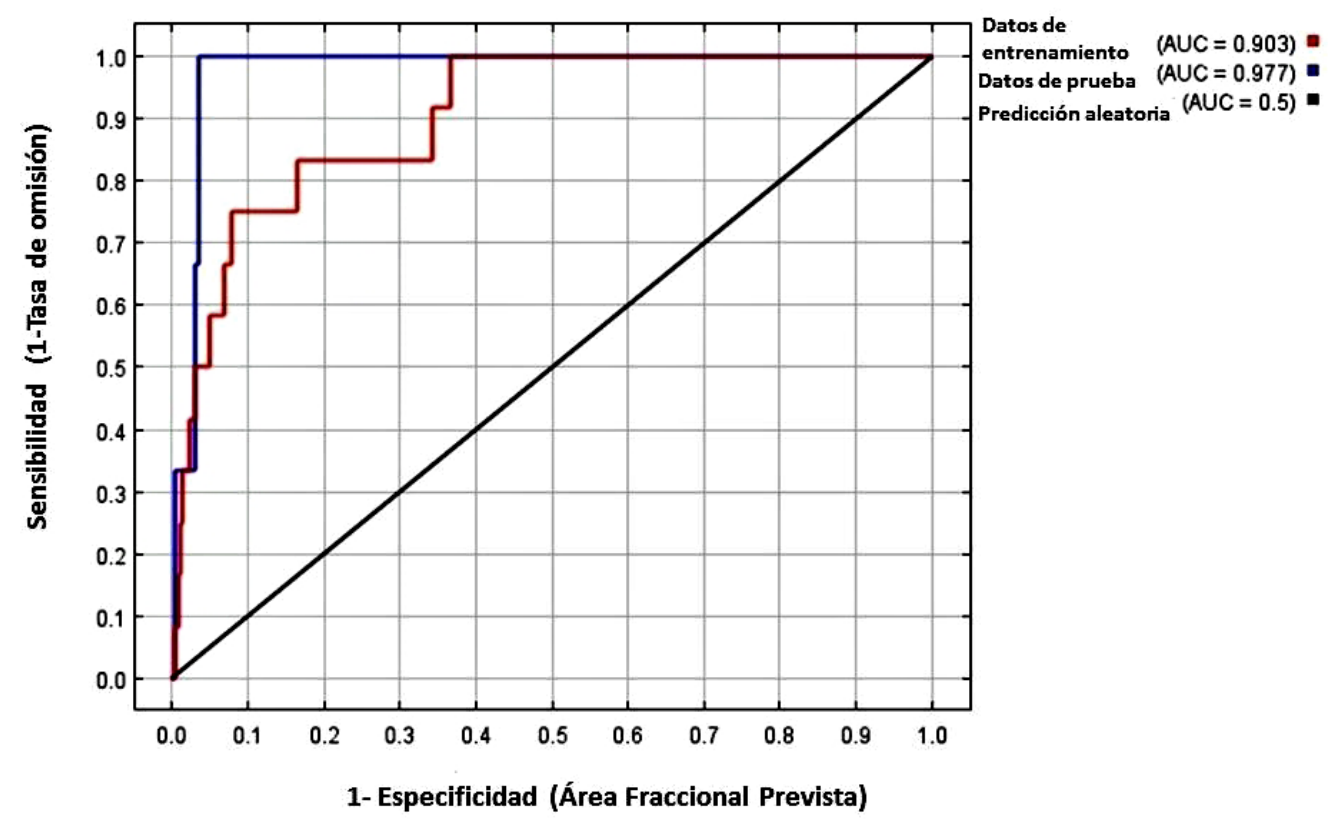

Figura 5. La prueba estadística muestran que modelación para los registros de oso caballo son adecuados, ya que el grafico (AUC) del modelo están por encima del umbral con un valor de 0.903 .

Área de Omisión y Predicción para Myrmecophaga tridactyla 0



Figura 6. Gráfico donde se muestra que los registros de la modelación están por debajo de la tasa de omisión, mostrando que los sitios de ausencia es la predicha por el modelo en el mapa. 
Para la modelación de la distribución potencial del oso caballo, se utilizaron 21 registros de 14 localidades (ver tabla 2) para este análisis se usó el MaxEnt 3.3, que utiliza el sistema de algoritmo de Máxima Entropía (www. cs.princeton.edu/ schapire/maxent) el cual es un método que estima la probabilidad de distribución equitativa (Phillips et al., 2006), el cual combina 19 variables bioclimáticas (temperaturas, humedad, precipitación), tomados de la base de datos Worldclim (http:// www.worldclim.org/bioclim), la modelación produce mapas potenciales para el hábitat de la especie (Phillips et al., 2006). Con esta información, se construyó un modelo de distribución que toma en cuenta todos los registros y las variables bioclimáticas y predice potencialmente el nicho ecológico del oso caballo para Honduras. La proyección de los registros es en grados y minutos, tomando las 21 localidades por considerarse localidades únicas y diferentes individuos. El modelo se validó con 5 repeticiones, con un $20 \%$ de los registros como grupo de prueba del modelo y un $80 \%$ de los registros restantes para la elaboración del modelo de distribución potencial. Se utilizó la opción básica del MaxEnt para la modelación.

\section{RESULTADOS}

De los cinco resultados de la modelación para el M. tridactyla se seleccionó la que presento mayor sensibilidad del área bajo la curva (AUC) siendo 0.903 la cual representa un modelo aceptable en la correlación sitios y variables. Así mismo la prueba de omisión cuyos resultados se muestran por debajo de la regresión logística en el gráfico (Figuras 5 y 6 ). El mapa producido por la modelación muestra menos probabilidad de distribución potencial en la región del Caribe hondureño siendo PN
Jeannette Kawas, Refugio de Vida Silvestre (RVS) de Texiguat, PN Pico Bonito, Jardín Botánico de Lancetilla, RVS de Cuero y Salado, PN Nombre de Dios y el PN Capiro y Calentura y las áreas protegidas de la RB Tawahaka y el PN Patuca El mapa de idoneidad de hábitat para el M. tridactyla estaría más distribuido hacia las áreas de la Reserva de la Biosfera del Río Plátano, los territorios Indígenas de Rus Rus, Warunta y Mocorón (Figura 4).

\section{DISCUSIÓN}

El mapa propuesto por Porini et al. (2004) presenta que los limites más septentrional es la costa Caribe de Guatemala y presenta de forma homogénea una distribución en todo el territorio de Honduras, El Salvador, Nicaragua y Costa Rica y una franja que cubre la parte media de Panamá hacia la costa del Caribe. En la mayoría de la literatura esta distribución es utilizada como referencia; sin embargo, los registros muestran que para Honduras se encuentra para el Caribe de manera histórica y para la región de la Moskitia los registros actuales.

Históricamente de acuerdo a los registros documentados de Marineros y Martínez 1998, la distribución del oso caballo se dio en la costa Caribe de Honduras estando muy asociado a las masas boscosas continuas de bosque lluvioso tropical de tierras bajas del Caribe hondureño, sin embargo las diferentes presiones antropogénicas a la que ha estado sometida la región desde los años 1900, por actividades agrícolas para la exportación de bananos, piñas, cítricos, palma africana (Laínez y Meza, 1973) entre otros han afectado el hábitat del M. tridactyla, según Portillo et al., 2010, llegando a la extirpación regional de la especies, sin embargo de acuerdo al mapa de distribución poten- 
cial producido por el MaxEnt basado en la gama de colores, de 0 a 1 la región del Caribe hondureño aún muestra posibilidades bioclimáticas potenciales (0.31-0.62) para su distribución (Figura 4).

Para la región de la Moskitia el mapa potencial de la presencia de la especie está asociado a las formaciones ecológicas siguientes: bosque siempre verde latifoliado de tierras bajas, bosque siempre verde latifoliado de tierras bajas en colinas cársticas onduladas, sabanas de graminoides cortos inundables con árboles latifoliado siempre verdes, sabanas de graminoides cortos con árboles aciculifoliados y carrizal pantanoso de agua dulce (Mejía y House, 2002).

Los listados oficiales actualizados de mamíferos para Guatemala, Belice, El Salvador y Costa Rica, confirman que el registro del oso caballo para Centro américa es solamente para los países de Honduras y Nicaragua, específicamente en la región de la Moskitia Hondureña y Nicaragüense en los años 2008, 2009 y 2012.

La ausencia de registros de $M$. tridactyla en los países de Guatemala, Belice y El Salvador con llevan asumir la no presencia del oso caballo en su distribución para estos países (McCarthy, 1998; McCarthy et al., 2006; Owens y Girón, 2012), sumado a esto la no detección en la región Caribe y los registros actuales de la especie en la Moskitia hondureña, llevan a proponer que el limite más al norte de distribución para el oso caballo es Honduras y específicamente la región de la Moskitia hondureña.

Las presiones actuales para la región de la Moskitia como el avance de la frontera agrícola, la ganadería extensiva, la cacería deportiva y el tráfico de especies, son las amenazas más altas por resolver (Secaira, 2013) y de esta forma mantener el hábitat óptimo para la especie, sin embargo, es poco lo que se conoce de su ecología y biología, su población, sus rangos hogareños y otros estudios que contribuyan a proponer estrategias de conservación para la especie y su hábitat.

Es alarmante que en un periodo de 40 años la especie haya sido extirpada de la costa Caribe de Honduras, y que las condiciones de conservación de las áreas protegidas de la Moskitia no estén garantizando la presencia de esta especie tal como se muestra en el mapa de la modelación potencial del oso caballo, donde se puede apreciar en sitios de registro histórico donde actualmente las condiciones bioclimáticas no son las más óptimas para la especie, esto atribuido a la pérdida del hábitat como consecuencia del avance de la frontera agrícola y cacería.

La Moskitia Hondureña pese a estar bajo una fuerte presión aún muestra una alta resilencia, ya que aún mantiene poblaciones de jaguares (Panthera onca), dantos (Tapirus bairdii), jagüillas (Tayassu pecari), venados cola blanca y tilopo (Odocoileus virginianus y Mazama temama) (Portillo y Hernández, 2011; Gonthier y Castañeda, 2013; Portillo y Elvir, 2013) entre otras especies claves en la estructura de las poblaciones de mamíferos grandes y medianos, además de ser el continuo boscoso más extenso en el país y parte fundamental en el funcionamiento del Sistema Nacional de Áreas Protegidas en Honduras y Nicaragua, por lo que se debe de considerar de alta prioridad su conservación. Es de urgencia la presencia de las instituciones responsables de la protección, conservación y el manejo responsable de la riqueza de biodiversidad en la región de la Moskitia hondureña (Secretaría de Recursos Naturales y Ambiente, Instituto de Conservación Forestal, Fis- 
calía del ambiente, ejército y la policía) ya que de no ser así, las ultimas masas boscosas estarán en riesgo a voluntad de innumerables colonos que cada año llegan en la búsqueda de nuevas tierras, destruyendo y transformando los continuos boscosos más extensos en Honduras.

\section{AGRADECIMIENTOS}

Se agradece a las organizaciones como AID/MIRA, Wildlife Conservation Society, Fundación PANTHERA, CANATUHR, RECOTUR, Proyecto Moskitia/PNud e Instituto de Conservación Forestal (ICF) que permitieron el uso de sus informes y que por su esfuerzo ha aportado información valiosa para la conservación de los mamíferos terrestres en Honduras. A los para-técnicos locales que con su dedicación y disciplina se han logrado obtener estos registros. A los biólogos hondureños dedicados al trabajo de campo y que con su labor silenciosa han contribuido al conocimiento y conservación de la biodiversidad del país. A Alexander Gonzales que dio su vida por la protección de la Moskitia. A la comunidad de Mabita, en la Moskitia hondureña como ejemplos a seguir en conservación. A Tomas Manzanares que arriesga su vida y aún sigue luchando por la protección y la conservación de los territorios indígenas miskitos. A ellos con especial aprecio.

\section{BIBLIOGRAFÍA}

\begin{abstract}
Alston, E.R. 1879-1882. Biologia Centrali-Americana Mammalia. Taylor and Francis, London.
\end{abstract}

Castañeda, F.E. 2007. Monitoreo biológico en la Biosfera de Río Plátano. DAPVS, AFECOHDEFOR, Mejorando Nuestra Herencia de UNESCO. Tegucigalpa. 128p.

Castañeda, F.E. 2008a. Monitoreo Biológico en la Reserva del Hombre y Biosfera de Río Plátano. DAPVS-AfECOHDEFOR, UNESCO, UQ. Tegucigalpa. $107 \mathrm{p}$.

Castañeda, F. 2008b. Situación Actual del Jaguar (Panthera onca) en la Reserva del Hombre y Biosfera del Río Plátano. wcs, AFE-COHDEFOR. Tegucigalpa. $19 \mathrm{p}$.

Castañeda, F., Herrera, L. y S. Pereira. 2013a. Muestreo Preliminar del jaguar (Panthera onca) y sus presas en el Parque Nacional Patuca. Reporte. Panthera/HelvetasHonduras/ Asociacion Patuca. Tegucigalpa. 12p.

Castañeda, F.E., J.R. McCranie y L.A. Herrera. 2013b. Staurotypus triporcatus (giant musk turtle, guao do tres filas) predation. Herpetological Review, 44(2):309.

Castañeda, F.E., L.A. Herrera y S. Pereira. 2013c. Behavior of two male jaguar scavenging on a marine dolphin in Honduras. Catnews, 58:312.

DAPVS (Departamento de Áreas Protegidas y Vida Silvestre). 2005. Actualización del Plan Estratégico del Sistema Nacional de Áreas Protegidas de Honduras (SINAPH). 2006 - 2015. Proyecto Biodiversidad y Áreas Protegidas (PROBAP). Tegucigalpa Honduras. 75 p.

Diniz, M y Brito, D. 2012. The Charismatic Giant Anteater (Myrmecophaga tridactyla): a Famous John Doe. Edentata, 13:7683

Estrada, N. 2006. Estudio del danto (Tapirus bairdii) en el Parque Nacional Sierra de Agalta, Olancho, Honduras. International Resources Group, Washington. 38 p.

ESNACIFOR (Escuela Nacional de Ciencias Forestales y Jardín Botánico y Centro de Investigación Lancetilla). 2012. Los Mamíferos de la Bahía de Tela y Montaña de Texiguat, Honduras. Pp 29-60, en: Investigaciones realizadas mediante subvención 13-2008 Procorredor.

Emmons, L. y H.F. Feer. 1997. Neotropical rainforest mammals: a field guide. Second edition. University of Chicago Press,
Chicago.

Gardner, A.L. 1993. Order Xenarthra. Pages 63-68, en: Mammal species of the world: a taxonomic and geographic reference (Wilson D.E. y D.M. Reeder, editors). Smithsonian Institution Press, Washington, D.C.

Goodwin, G.G. 1942. Mammals of Honduras. Bulletin of the American Museum of Natural History, 79:107195.

Gonthier, D.J. y F.E., Castañeda. 2013. Large- and medium-sized mammal survey using camera traps in the Sikre River in the Río Plátano Biosphere Reserve, Honduras. Tropical Conservation Science, 6(4):584-591.

Hall, E.R. 1981. The mammals of North America. Second edition. John Wiley \& Sons, New York.

Handley, C.O., JR. 1950. A Fish and Wildlife Survey of Guatemala. Special Scientific Report. Wildlife, 5. United States Department of Interior Fish and Wildlife Service.

Holdridge, L. 1971. Ecología basada en zonas de vida. Instituto Interamericano de Ciencias Agrícolas. IICA. 216 p.

IUCN. 2004. Red list of threatened species. Gland, Switzerland: International Union for Conservation of Nature and Natural Resources.

Koster, J.M. 2008. Giant anteater (Myrmecophaga tridactyla) killed by hunters with dogs in the Bosawas Biosphere Reserve, Nicaragua. Southwestern Naturalist, 55:414416.

Marineros L. y F. Martínez. 1998. Guía de campo de los mamíferos de Honduras. Instituto Nacional de Ambiente y Desarrollo, Tegucigalpa, Honduras.

Mejía, T.M. y P.R., House. 2002. Mapa de ecosistemas vegetal de Honduras. Manual de Consulta. Proyecto de Administración de áreas rurales (PAAR), SAG, Banco Mundial, AFE-COHDEFOR. Tegucigalpa, M.D.C., Honduras.

McCarthy, T.J. 1998. Mammals of Belize: A Checklist. Producciones de la Hamaca Caye Caulker, Belize, $19 \mathrm{pp}$.

McCarthy, T.J. y S., Pérez. 2006. Land and freshwater mammals of Guatemala: Faunal Documentation and Diversity Pp. 674, en: Biodiversidad de Guatemala. (Cano, E.B., ed.). Universidad del 
Valle de Guatemala, Guatemala.

McCain, C.M. 2001. First evidence of the giant anteater (Myrmecophaga tridactyla) in Honduras. Southwestern Naturalist, 46:252254.

Laínez, V. y V., Meza. 1973. El Enclave Bananero en Honduras. Nueva Sociedad. NRo. 6 de mayo-junio. pp 21-43.

Nowak, R.M. 1999. Walkers' mammals of the world, Sixth edition, Volume 1. Johns Hopkins University Press, Baltimore, Maryland.

Owens, J., y L., Girón. 2012. Revised Checklist and Distribution of Lands of Mammals of El Salvador. Natural Science Research Laboratory, 310: 30Pp.

Portillo, H., C. Zelaya y M. Vásquez. 2006. Ecología de la sub-población de jaguar (Panthera onca) en el parque nacional Pico Bonito $y$ el refugio de vida silvestre Texiguat. International Resources Group, Washington. 38 p.

Portillo, H., T. Manzanares, T. Manzanares Jr., S. Lacut y R. Lacut. 2008. Estimating jaguar population using traps camera in one hundred square kilometers in Rus Rus La Mosquitia, Honduras. Wildlife Conservation Society. Tegucigalpa. 20 p.

Portillo, H. y M. Vásquez. 2009. Expedición y evaluación de la Reserva de la Biosfera Tawahka, La Mosquitia, Honduras. wcs-ICF. 24 p.
Portillo, H.O., Matamoros W.A. y S.L., Glowinski. 2010. Distribution and conservation status of the giant anteater (Myrmecophaga tridactyla) in Honduras. Southwestern Naturalist, 55:119-121.

Portillo-Reyes H y J., Hernández. 2011. Densidad del jaguar (Panthera onca) en Honduras: primer estudio con trampas-cámara en La Mosquitia hondureña. Revista Latinoamericana de Conservación, 2(1):45-50

Portillo, H. 2013. Informe del monitoreo de mamíferos en el Refugio de Vida Silvestre de Cuero y Salado usando trampas cámara como método de muestreo en el Sendero de las Bujajas. Bosques del Mundo, RECOTURH, FUCSA, CANATURH, FUPNAPIB. Tegucigalpa, $28 \mathrm{pp}$

Portillo, H. y Elvir, F. 2013. Composición, estructura y diversidad de los mamíferos terrestres grandes y medianos en 16 Áreas Protegidas en Honduras, usando fotocapturas como evidencia de registro. Mesoamericana, 17(2):15-31.

Portillo, H. 2013. Segundo Informe del Proyecto: Monitoreo biológico para establecer la línea base del sistema lagunar de Karatasca, de los mamíferos terrestres y guara roja en Rus Rus en La Moskitia hondureña. PNUD, INCEBIO, ICF,. Tegucigalpa M.D.C., Honduras. Pp 31.

Porini, G. Ryland y A.R., Samudio. 2004. The 2004 Edentate species assesmment workshop. Species Summary. Edentata,6:1-26.

Phillips, S.J., Anderson, R.P. y R.E., Schapire. 2006. Modelling Distribution and Abundance with Presence Only-Data. Journal of Applied Ecology, 43, 405-412.

PROBAP/AFE-CODEHFOR. 2005.Elmonitoreo biológico del SINAPH. Documentos del proyecto de biodiversidad en Áreas Prioritarias. Informe técnico. PROBAP/AFE-CODEHFOR. Tegucigalpa. Honduras. 75 pp.

Reid, F.A. 1997. A field guide to the mammals of Central America and southeast Mexico. Oxford University Press, New York.

Secaira, E. 2013. Análisis y síntesis de los 10 planes de conservación elaborados para las Áreas Protegidas de trabajo del proyecto ProParque. usaid ProParque.

Timm, R.M. y R.K. Laval. 2000. Mammals. Pp. 223-244, 553-560, en: Monteverde: Ecology and conservation of a tropical cloud forest (Nadkarni, N.M. and N.T. Wheelwright, eds.). Oxford University Press, New York.

Wetzel, R.M. 1985. The identification and distribution of Recent Xenathra (Edentata). Pp. 52-57, en: Mammal species of the world. (Honacki, J.H., K.E. Kinnan, y J. W. Koeppl, eds.). Allen Press and Association of Systematics Collection, Lawrence, Kansas. 